\title{
RELACIONES ENTRE LOS FENÓMENOS GEOGRÁFICOS Y LA CONCIENCIA GEOGRÁFICA EN TACNA
}

María Morris Ayca', Oscar Panty Neyra ${ }^{2}$

\author{
R E S U M E N
}

\begin{abstract}
El presente trabajo se orienta al análisis de las caracteristicas geomorfologicas yclimáticas del espacio geográfico racneño, y la forma como éstas se relacionan, dando lugar a una diferenciación de regiones, zonas y subzonas geográficas, que establecen determinadas condiciones para el comportamiento del hombre que construye y explota el espacio que socialmente ocupa.
\end{abstract}

Se destaca la importancia que tiene el conocimiento cientifico de la realidad espacial para el desarrollo de la conciencia geográfica, entendiendo a ésta como la actitud positiva y la identificación del hombre con el espacio geografico, sus recursos, potencialidades y situaciones medioambientales.

En consecuencia, el desarrollo y afianzamiento de la conciencia geográfica puede y debe contribuir al mejoramiento y progreso de las condiciones de vida de la poblacion regional.

A B S TR A C T

This work focuses on the analysis of geomorphological and climatic characteristics of the city of Tacna; and on the way these factors relate each other with the consequent geographical variety of regions, zones and subzones; and determinant conditions affecting population's behaviour people, in charge of building and exploting the land where he/she lives.

It is emphasized the importance of scientific knowledge of reality to develop geographical awareness; that is, the positive attitude and identification of human being with his/her local geography, resources, and enviromental perspectives and situations.

Finally, we conclude stating that both development and geographical consciousness can and must buil dimproved conditions of living for the regional population.

\section{INTRODUCCIÓN}

Discernir sobre el significado del conocimiento geográfico en la región o en cualquier lugar del espacio terrestre, aun en el espacio cósmico, implica asumir una definida actitud cognitiva-estimativa de lo que es el espacio como fuente y medio de vida y de como este espacio debe continuar sustentando, equilibradamente, la cadena de la vida en todas sus expresiones, entre ellas la humana.

\footnotetext{
1. Licenciada en educación.

2. Licenciado en educación.
}

El conocimiento geográfico empieza con el hombre que aparece en la tierra y se relaciona con su entorno para subsistir hace dos millones de años. Hoy como en el alba de la humanidad, este conocimiento elevado al nivel científico, puntualiza la existencia humana (principio geográfico fundamental) lo que significa determinar los aspectos de extensión, delimitación y diferenciación con otras áreas o regiones, pero al mismo tiempo determinar sus relaciones y correlaciones regionales.

Cada espacio regional ofrece a sus respectivas poblaciones un conjunto de posibilidades cuyo aprovechamiento requiere de un conocimiento 
geográfico integral que debe empezar registrando las condiciones ambientales: El territorio, con sus características geomorfológicas, climáticas, divisiones y subdivisiones regionales. Los recursos disponibles y potenciales, como suelos, aguas, minerales, flora, y fauna, además la problemática de los riesgos naturales.

En otro orden de cosas, el conocimiento geográfico debe abordar los procesos de ocupación y organización de los espacios que el hombre habita, explota, administra y usufructúa, constituyendo colectividades locales de base, pueblos, empresas, mercados, sistemas de comunicación, divisiones administrativas, Estado territorial. Asimismo, la organización del espacio y el comportamiento social, la estructura política e ideológica, la tradición regional. En la condición de región fronteriza con otros Estados, la situación geopolítica.

En consecuencia, el conocimiento geográfico en la región debe contribuir a la toma de conciencia de la realidad espacial, sus recursos y potencialidades de desarrollo, pero también a la toma de conciencia del comportamiento de la población que ocupa y organiza el espacio a partir de determinados presupuestos ideológicos, políticos y culturales.

\section{DIVISIÓN DEL ESPACIO GEOGRÁFICO}

\subsection{CRITERIOS}

Tradicionalmente, la región geográfica fue comprendida como un área homogénea, con sus características físicas y naturales que la diferenciaban de las áreas vecinas. Los geógrafos entendian por región a cualquier área grande más o menos definida espacialmente por ciertos rasgos naturales, o un área donde las condiciones físicas son homogéneas. No faltaron quienes sostuvieron enfáticamente que la realidad natural es una sola, y que por tanto la región como división de esta realidad sólo existe en el pensamiento. La región es un constructo mental de los estudiosos. Pero el desarrollo de las investigaciones geográficas demandó la necesaria regionalización del espacio para su mejor estudio. De aqui que en los tiempos modernos se haya ensayado nuevas definiciones cuya visión de sintesis nos ofrecen Córdova y Bernex:

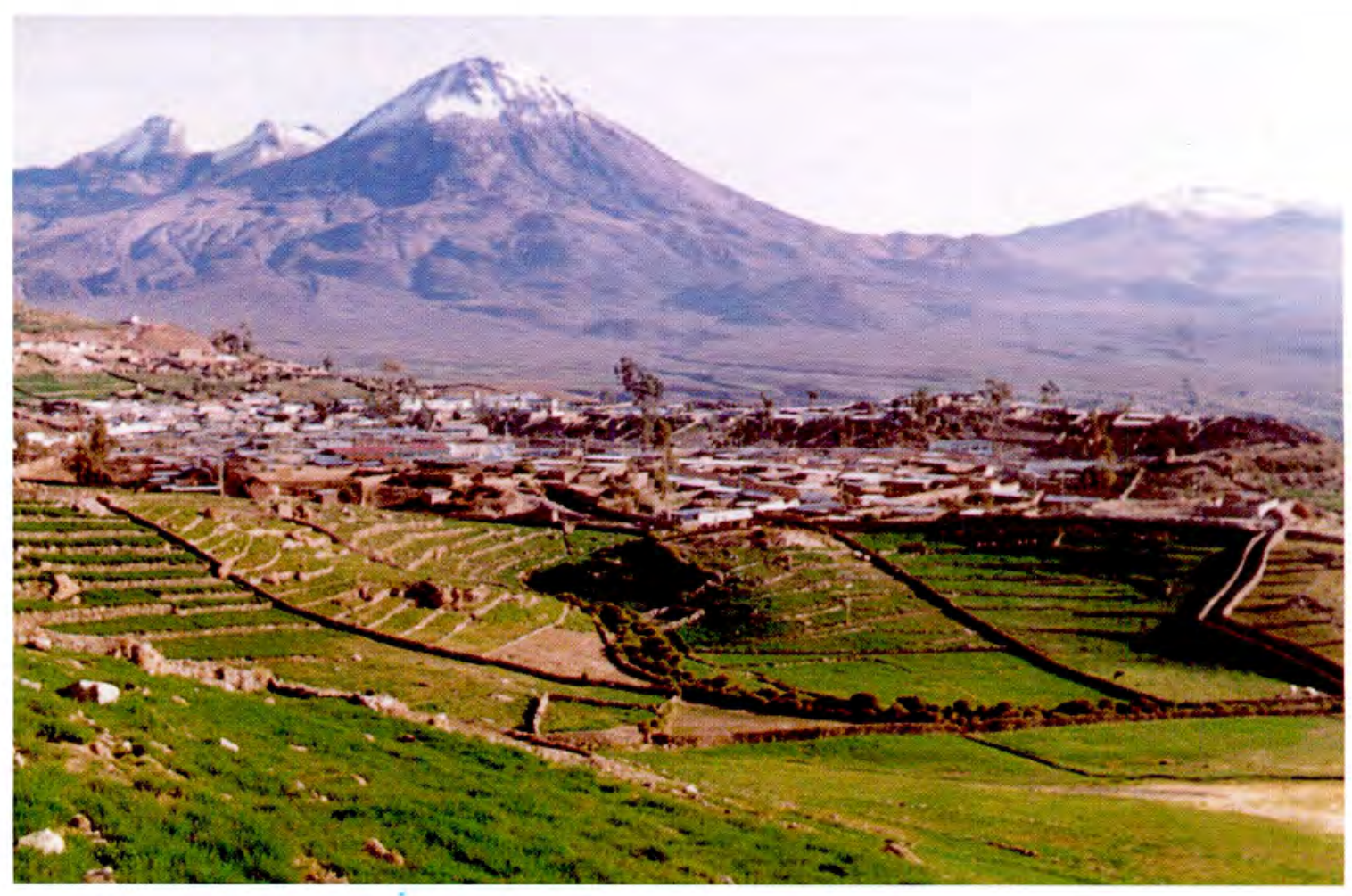

Región Andina, zona interandina, subzona: valle alto de Candarave. Vista de Candarave al pie del volcán Yucamani. 
En la concepción geográfica moderna, la región es a) una porción de espacio equilibrado. Ella representa una especie de óptimo de la organización espacial, dentro de la cual se efectúa la integración ciudad-campo en una armonía más o menos perfecta. b) Es una porción de espacio organizado que disfruta de cierta permanencia capaz de resistir cambios, y por tanto, es una entidad real. c) Es una porción de espacio original, único, cuyos fenómenos por su existencia misma o por esencia poseen ventajas de ubicación con respecto a otros espacios. (Córdova y Bernex, 1983)

Pero los geógrafos han advertido que los hechos o fenómenos se encuentran interrelacionados de tal modo que lo natural se combina con lo humano, resultando compleja la distinción de regiones en base a una homogeneidad visible en sus elementos. Entonces, el problema que se plantea es cómo las regiones se delimitan en el marco de la disposición de los elementos naturales y de cómo las consideraciones humanas están presentes en tal delimitación. No es suficiente la cercanía a los elementos geográficos, sino también la capacidad de percepción de los elementos que se combinan y se yuxtaponen. Aqui es donde surge el problema de las concepciones geográficas.

... el estudio regional no se queda en un simple catálogo de rasgos, sino que pasa al análisis del sistema de interacción. Como bien dice Vance (1976) los estudios regionales pueden ser situados en una escala ascendente de complejidad, según el nivel de integración de las variables. En primer lugar, las regiones pueden delimitarse sobre la base de una variable, como por ejemplo, la integración de las zonas de mercado y de distribución al por mayor. En zonas de mercado y de distribución al por mayor. En segundo lugar, las regiones pueden delimitarse sobre la base de un complejo de variables conexas, acercándose más a la realidad territorial-cultural. En tercer lugar, se encuentra el estudio regional complejo que se realiza desde una perspectiva histórica y cuyo objeto es mostrar la unidad funcional de los rasgos culturales, económicos y sociales de la región." (Córdova y Bernex, 1983).

Lo cierto es que, como recalcan Córdova y Bernex, una región es un sistema abierto y extremadamente complejo, donde un elemento "X" puede comportarse como parte de una región natural, como también parte de una región histórico-cultural, de una región socio-económica, politica y/o administrativa.

Veamos a continuación los criterios que se han tomado en cuenta para la delimitación del espacio geográfico en regiones y sus correspondientes subdivisiones:

a) Geomorfológicos, los que dan referencia a las características de relieve con lo que se tiene la división Costa, Sierra y Selva.

b) Climáticos, los que destacan la importancia del factor climático.

c) Geoquímicos, los que otorgan significación a los componentes del suelo y subsuelo.

d) Hidrológicos, los que dan preponderancia a la distribución y curso de las aguas superficiales.

e) Biogeográficos, los que dan preferencia a la flora y fauna que forman complejos sistemas de asociaciones y formaciones.

f) Antropogeográficos, los que juzgan la adaptación y residencia de los hombres que construyen sus espacios.

g) Integralistas, los que consideran todos los elementos componentes del espacio geográfico en acción e interrelación múltiple.

Nos inclinamos por el criterio de la interrelación de todos los componentes del espacio que por su proximidad y frecuencia delimitan áreas de organización diferenciadas, las que a su vez son objeto de ocupación y modificación por acción social del hombre.

\subsection{DIVISIÓN DEL ESPACIO GEOGRÁFICO DE TACNA}

Los estudios acerca de la realidad geográfica de Tacna han conducido a plantear que, de acuerdo a los condicionamientos que presentan el relieve, el clima y los recursos naturales, sus regiones geográficas, Costa y Andina, van diferenciando en su interior zonas y subzonas con caracteristicas muy peculiares, las cuales presentamos a continuación:

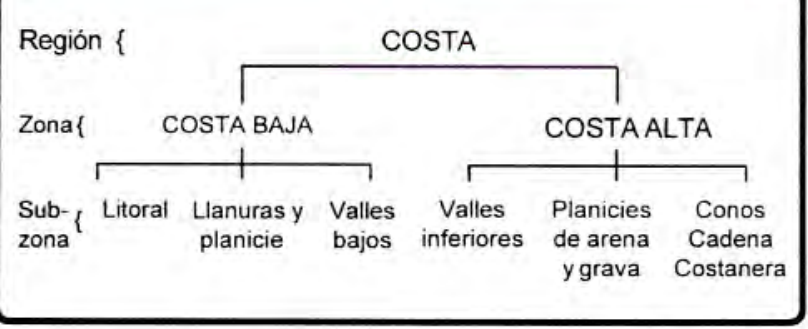




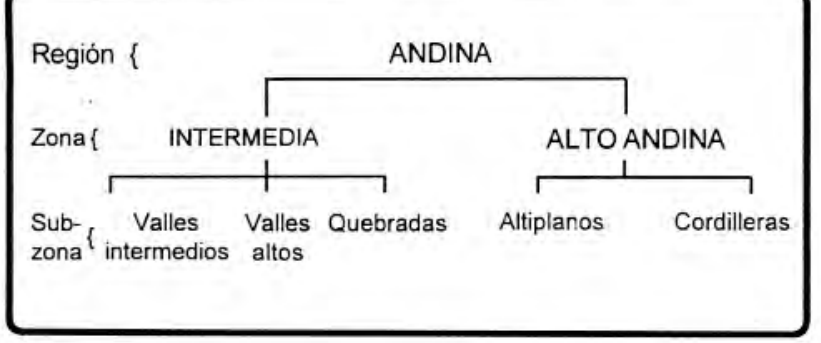

En el trabajo Introducción al estudio de las caracteristicas geomorfólogicas y climáticas de Tacna y su relación con la conciencia geográfica, describimos y explicamos el comportamiento de los elementos componentes de las regiones geográficas, teniendo en cuenta que el estudio objetivo e integral de las regiones no se puede realizar de manera aislada, sino en permanente interrelacion o influencia reciproca. Para tal caso se ha establecido relaciones entre las variables identificadas como: Características geomorfológicas y características climáticas; recursos naturales y conciencia geográfica; ocupación espacial y riesgos naturales: riesgos naturales y conciencia geográfica;

Caracteristicas geomorfológicas y climáticas en relación a la conciencia geográfica.

\section{RELACIÓN ENTRE LAS VARIABLES DEL ESPACIO REGIONAL}

\subsection{RELACIÓN ENTRE LAS CARACTERÍSTICAS GEOMORFOLÓGICAS Y CLIMÁTICAS DE TACNA:}

Los estudios diversos sobre el comportamiento de los elementos y factores climáticos nos dan a conocer que la relación que existe entre estos y las geoformas del espacio, materia de estudio, genera determinadas condiciones ambientales. Es así que la forma y composición del suelo ińfluye en el comportamiento de la temperatura, lo que podemos verificar en el área espacial de los valles inferiores de Caplina, Sama, Locumba, valles altos de la zona interandina y la zona altoandina que a continuación comentamos.

El valle inferior del Caplina que se extiende aproximadamente desde los 1080 msnm (Pachía), tiene la forma de un abanico que se proyecta hacia el litoral, flanqueado por dos cerros de una elevación promedio de $800 \mathrm{msnm}$, cuya composición es de roca volcánica, cubierta de arena fina por acción eólica, situación caracteristica que facilita la captación solar en el dia y que se enfría más rápidamente en la noche, haciendo que la temperatura media máxima tomada en Calana sea de $22.7^{\circ} \mathrm{C}$, y la temperatura media mínima sea de $10.82^{\circ} \mathrm{C}$.

Mientras que la temperatura máxima absoluta en los últimos 30 años ha llegado hasta los $32^{\circ} \mathrm{C}$. y ésta se produce entre las 13 y 14 horas del dia. Por otra parte, la temperatura mínima absoluta que se produce generalmente por la noche llega hasta los $0.0^{\circ} \mathrm{C}$ en el mes de julio.

La amplia cobertura del valle inferior en ensanchamiento creciente, permite mayor circulación de los vientos que transportan masas de aire húmedo y helado hacia el interior del valle.

La combinación de la geoforma con la composición de los suelos, la relativa radiación solar interferida por el desplazamiento de las masas de aire húmedo y la nubosidad persistente, determinan un tipo de clima árido, lo que a su vez restringe la biodiversidad de la flora y fauna.

Otra es la situación del valle de Sama, que se proyecta como una franja estrecha flanqueada por dos cerros de poca elevación. La composición de los suelos del área circundante corresponde a depósitos aluviales, predominando la grava, arena gruesa y cantos rodados, lo que posibilita que el grado de retención térmica-solar sea mayor en el dia respecto al valle inferior de Tacna. En los últimos 30 años, la temperatura media máxima fue de $24.8^{\circ} \mathrm{C}$. La temperatura media mínima de $12.9^{\circ} \mathrm{C}$, llegando la temperatura máxima absoluta por sobre los 30 y $32^{\circ} \mathrm{C}$; mientras que la temperatura mínima absoluta desciende hasta los 2.2 y $5.2^{\circ} \mathrm{C}$, siendo la temperatura media extrema de $18.8^{\circ} \mathrm{C}$.

En consecuencia, la combinación de estos elementos determina un clima con temperaturas medias ligeramente mayores que la de Tacna, dando lugar a un clima templado.

En Locumba encontramos a un valle inferior con cauce más angosto que el de Sama, bordeado por cerros correspondientes a la Formación Moqueguana y formación geológica sedimentaria de tipo continental, en cuyos cerros predominan suelos arcillosos, limosos y de grano medio.

Esta composición da lugar a que la captación de la radiación solar sea más lenta y el 


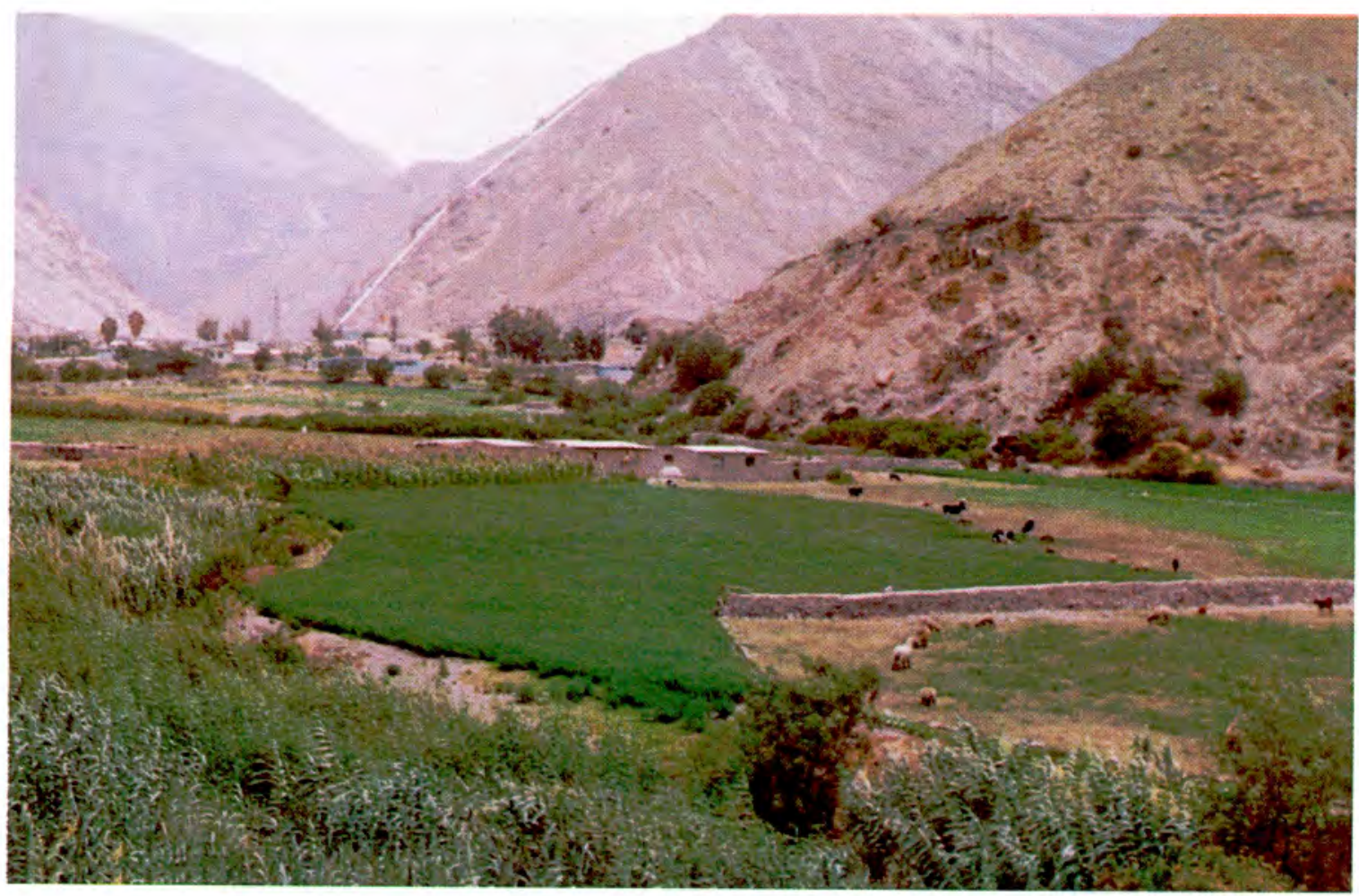

Región costa, zona costa alta, subzona: valle inferior en el limite con la zona interandina. Vista del valle de Locumba en el sector de Chintari.

enfriamiento sea también más lento, haciendo que la retención del calor sea por tiempo más prolongado que en los valles que venimos citando.

De conformidad con las informaciones registradas en el centro regional del SENAMHI, la temperatura media máxima es de $25.9^{\circ} \mathrm{C}$, mayor que la del valle Sama y Tacna. La temperatura media minima de $12.3^{\circ} \mathrm{C}$, mientras que la temperatura máxima absoluta asciende por encima de $33.8^{\circ} \mathrm{C}$ hasta los $35^{\circ} \mathrm{C}$, siendo la temperatura media de $19.4^{\circ} \mathrm{C}$.

La precipitación en este sector es nula, lo que difiere de las precipitaciones registradas en Tacna y Sama durante los últimos 30 años.

El conjunto de estas condiciones determinan un clima árido y cálido.

El valle alto de Tarata, ubicado a 3,070 msnm, se caracteriza por ser muy accidentado, donde destacan laderas y quebradas compuestas de rocas volcánicas sedimentarias, correspondientes a la formación Tarata. Los suelos son predominantemente claros, incluyendo bancos de tufos volcánicos en su miembro inferior de color marrón y en el miembro superior de color rojizo. Esta composición da lugar a que la captación de la radiación solar sea más lenta en el día y asimismo, demore su enfriamiento por la noche.

En consecuencia, las características del relieve unificado a los factores climáticos, como la altitud, determinan el comportamiento del clima, obteniendo una temperatura media máxima de $18.78{ }^{\circ} \mathrm{C}$ y una media mínima de $5.11^{\circ} \mathrm{C}$. La temperatura máxima absoluta llega hasta los $28^{\circ} \mathrm{C}$ en el mes de agosto y la temperatura mínima absoluta desciende hasta $-2.2^{\circ} \mathrm{C}$ en el mes de agosto.

El valle alto de Candarave, ubicado a 3415 msnm, se caracteriza por tener un relieve accidentado en el que se aprecia predominantemente áreas más abiertas. La composición de los suelos de origen volcánico sedimentario y la existencia de depósitos aluviales relacionados con las geoformas del lugar y el efecto del clima, como son la altitud, la velocidad 
del viento, el comportamiento de la presión atmosférica y la escasa humedad, hacen que la temperatura media máxima oscile entre $15.38^{\circ} \mathrm{C}$ y $16^{\circ} \mathrm{C}$. La temperatura media minima tiene un promedio de $3.56^{\circ} \mathrm{C}$, mientras que la temperatura máxima absoluta llega hasta los $25^{\circ} \mathrm{C}$ en el mes de julio y la temperatura minima absoluta desciende hasta $-2.0^{\circ} \mathrm{C}$.

\subsection{RELACIÓN ENTRE RECURSOS NATURALES Y CONCIENCIA GEOGRÁFICA}

Se constata que los recursos naturales son escasos como los de naturaleza hidrica e hidrobiológica; o son poco diversificados como la flora y fauna; o es inadecuado su uso como el caso de los suelos; o son afectos a la depredación como ocurre en Playa Inglesa, o no cuentan con la debida asistencia especializada para su conservación y aprovechamiento, como sucede en la zona altoandina con los bofedales y la flora y fauna dependiente.

En la población departamental, en sus diversos sectores y estratos (comunidades de base, autoridades, etc), se carece de una clara comprensión de lo que significa la conservación y aprovechamiento sostenido y mejorado de los recursos naturales, prueba de ello es que no existe una politica de conservación que responda a los planteamientos del organismo mundial denominado Unión para la Conservación de la Naturaleza y sus Recursos (UICN), expresados en términos siguientes: 1. Mantención de los procesos ecológicos esenciales y los sistemas de mantenimiento de la vida. 2. Preservación de la diversidad genética. 3 . Utilización sostenida de las especies y de los ecosistemas.

Si consideramos que la conciencia geográfica es la actitud positiva y la identificación del hombre con el espacio geográfico, sus recursos, potencialidades y las condiciones ambientales con las que se relaciona, entonces, es fundamental establecer la relación entre conciencia geográfica del poblador tacneño y los recursos naturales de la región.

Sólo en la medida que se tenga una conciencia geográfica en desarrollo, será posible la utilización optimizada de los recursos naturales, como por ejemplo, los recursos hídricos que en la agricultura deben ser aprovechados con nuevas técnicas, lo que permitiria la ampliación de la frontera agrícola. Del mismo modo, la relación conciencia geográfica-recursos hidrobiológicos permitiría un mejor aprovechamiento de los mismos, particularmente potenciando su poblamiento y repoblamiento de truchas en las fuentes de aguas continentales permanentes del departamento, para beneficio de las poblaciones ribereñas del interior.

Igualmente, establecer la relación conciencia geográfica-energia solar; conciencia geográficarecurso flora; conciencia geográfica-recurso fauna.

El desarrollo y afianzamiento de estas relaciones permitiria una mejor vinculación del hombre con su medio geográfico y en consecuencia, el mejoramiento y progreso de las condiciones de vida de la población regional.

\subsection{RELACIÓN OCUPACIÓN ESPACIAL Y RIESGOS NATURALES}

La ocupación espacial implica la organización del espacio que el hombre habita, explota, administra y usufructúa, constituyendo instituciones y formas de comportamiento social. Por otra parte, los riesgos naturales son probabilidades de ocurrencia de un fenómeno natural que pueda provocar pérdidas humanas y daños de sus bienes.

La población tacneña ocupa espacios en los que se puede distinguir zonas de riesgo de diversos grados: riesgos sísmicos, como por ejemplo en el cono norte de la ciudad de Tacna y la población de Chintari; riesgos torrenciales en las zonas de cauces de rios y quebradas.

Del mismo modo, donde se ubican asentamientos humanos de población numerosa como la ciudad de Tacna, sobre el cauce del río Caplina, el pueblo de Mirave ocupando todo lo ancho de la desembocadura de la quebrada del mismo nombre.

Igualmente, zonas de riesgo de deslizamiento de terrenos, como el área agricola de Pallata. Además, riesgos climáticos en las regiones interandinas y altoandinas (sequia, helada, lluvias excesivas).

La relación ocupación espacial-riesgos naturales plantea un conocimiento integral de la naturaleza del fenómeno y su frecuencia en el espacio geográfico, a fin de asumir actitudes de prevención, de previsión a fin de evitar y limitar los daños que se puedan generar. 


\subsection{RELACIÓN RIESGOS NATURALES Y CONCIENCIA GEOGRÁFICA}

El conocimiento de la relación anterior nos lleva a la toma de conciencia de los riesgos desde el nivel del núcleo familiar hasta los grupos humanos y entidades de mayor complejidad, tales como las comunidades de base, las empresas, el gobierno local y las instituciones del Estado.

La toma de conciencia de los riesgos implica un conocimiento del pasado y de la situación presente. Conocer, por ejemplo, la periodicidad del retorno de los fenómenos y tomar acciones de prevención ante probables ocurrencias basadas en el reconocimiento de signos anunciadores. La conciencia geográfica de los riesgos naturales es la clave de acciones futuras.

\subsection{RELACIÓN ENTRE LAS VARIABLES CARACTERISTICAS GEOMORFOLÓGICAS Y CLIMÁTICAS CON LA VARIABLE CONCIENCIA GEOGRÁFICA}

Las variables caracteristicas geomorfológicas entrañan un conocimiento de la realidad física del espacio, su naturaleza, localización, dinamicidad, relaciones. Las variables características climáticas permiten conocer los elementos condicionantes de la situación ambiental del espacio geográfico.

No es posible una adecuada ocupación y organización del espacio geográfico para el

\section{BIBLIOGRAFIA}

CORDOVA, Wildegardo y BERNEX, Nicole. Región geográfica, Regionalización y región para el desarrollo. En Regionalización, Proyecto, Debate y Posibilidad. Instituto Peruano de Estudios Geopolíticos, Estratégicos. Fundación EBERT. Lima 1983.

DOLLFUS, Oliver. Territorios andinos, reto y memoria. IEP Ediciones Lima 1991

JAEN, Hugo y ORTIZ, Guillermo. Geología de los cuadrángulos de la Yarada y Tacna. Boletín $N^{\circ} 06$. Comisión Carta Geológica Nacional - Lima 1993

JAEN LA TORRE, Hugo. Geologia del cuadrángulo de Tarata. Boletin N 11. Comisión Carta Geológica Nacional. Lima 1965. desarrollo optimizado de las actividades humanas si no se tiene como punto de partida el conocimiento de las bases físicas en las que se enmarca dicho espacio.

En este sentido, se trata de comprender y asumir una actitud participativa a partir del espacio geográfico que se ocupa y se construye, más aun en las condiciones de departamento fronterizo, como es Tacna, colindante con dos Estados nacionales.

\section{CONCLUSIONES}

- El estudio del espacio geográfico, su ocupación y organización social es básico para el planeamiento del desarrollo departamental en todos los niveles de la administración pública y privada.

- El compromiso y la participación de la población en el desarrollo regional, está en estrecha relación con el conocimiento integral del espacio geográfico de Tacna, a partir de las caracteristicas geomorfológicas y climáticas del espacio regionalizado.

- Es necesario desarrollar y afianzar la conciencia geográfica de la población del departamento en condiciones de frontera, a fin de potenciar la defensa, conservación y explotación optimizada de los recursos naturales, en función de los más altos intereses nacionales.

J. WILSON y W GARCIA. Geologia de los cuadrángulos de Pachía y Palca. Vol. II, N ${ }^{\circ}$ 04. Comisión Carta Geológica Nacional. Lima 1962

PANTY NEYRA, Oscar y MORRIS AYCA, Maria. Regiones Geográficas y climas de Tacna. Introducción al estudio de las características geomorfológicas y climáticas.. UNJBG, FACE. Tacna 1996.

BROCK EGG, Antonio. Ecología de un pais complejo, en Gran Geografía del Perú. Ed. Monfer. Juan Mejia Baca. Vol. II Lima 1987. 